\title{
Rabinizer 4: From LTL to Your Favourite Deterministic Automaton
}

\author{
Jan Křetínský(®) , Tobias Meggendorfer®, \\ Salomon Sickert (D), and Christopher Ziegler \\ Technical University of Munich, Munich, Germany \\ jan.kretinsky@gmail.com, \{meggendo, sickert\}@in.tum.de
}

\begin{abstract}
We present Rabinizer 4, a tool set for translating formulae of linear temporal logic to different types of deterministic $\omega$-automata. The tool set implements and optimizes several recent constructions, including the first implementation translating the frequency extension of LTL. Further, we provide a distribution of PRISM that links Rabinizer and offers model checking procedures for probabilistic systems that are not in the official PRISM distribution. Finally, we evaluate the performance and in cases with any previous implementations we show enhancements both in terms of the size of the automata and the computational time, due to algorithmic as well as implementation improvements.
\end{abstract}

\section{Introduction}

Automata-theoretic approach [VW86] is a key technique for verification and synthesis of systems with linear-time specifications, such as formulae of linear temporal logic (LTL) [Pnu77]. It proceeds in two steps: first, the formula is translated into a corresponding automaton; second, the product of the system and the automaton is further analyzed. The size of the automaton is important as it directly affects the size of the product and thus largely also the analysis time, particularly for deterministic automata and probabilistic model checking in a very direct proportion. For verification of non-deterministic systems, mostly non-deterministic Büchi automata (NBA) are used [EH00,SB00, GO01, GL02, BKŘS12,DLLF+16] since they are typically very small and easy to produce.

Probabilistic LTL model checking cannot profit directly from NBA. Even the qualitative question, whether a formula holds with probability 0 or 1 , requires automata with at least a restricted form of determinism. The prime example are the limit-deterministic (also called semi-deterministic) Büchi automata (LDBA) [CY88] and the generalized LDBA (LDGBA). However, for the general quantitative questions, where the probability of satisfaction is computed, general limitdeterminism is not sufficient. Instead, deterministic Rabin automata (DRA) have

This work has been partially supported by the Czech Science Foundation grant No. P202/12/G061 and the German Research Foundation (DFG) project KR 4890/1-1 "Verified Model Checkers" (317422601). A part of the frequency extension has been implemented within Google Summer of Code 2016.

(c) The Author(s) 2018

H. Chockler and G. Weissenbacher (Eds.): CAV 2018, LNCS 10981, pp. 567-577, 2018.

https://doi.org/10.1007/978-3-319-96145-3_30 


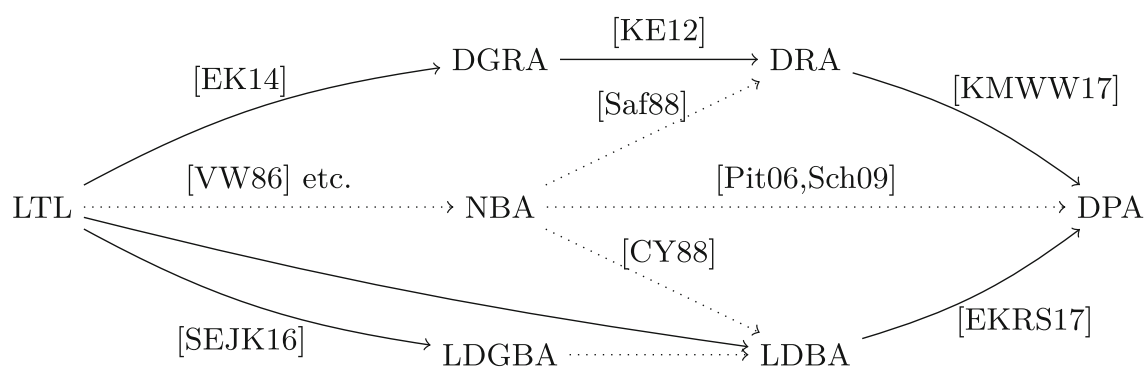

Fig. 1. LTL translations to different types of automata. Translations implemented in Rabinizer 4 are indicated with a solid line. The traditional approaches are depicted as dotted arrows. The determinization of NBA to DRA is implemented in ltl2dstar [Kle], to LDBA in Seminator [BDK+17] and to (mostly) DPA in spot [DLLF+16].

been mostly used [KNP11] and recently also deterministic generalized Rabin automata (DGRA) [CGK13]. In principle, all standard types of deterministic automata are applicable here except for deterministic Büchi automata (DBA), which are not as expressive as LTL. However, other types of automata, such as deterministic Muller and deterministic parity automata (DPA) are typically larger than DGRA in terms of acceptance condition or the state space, respectively. ${ }^{1}$ Recently, several approaches with specific LDBA were proved applicable to the quantitative setting [HLS+15, SEJK16] and competitive with DGRA. Besides, model checking MDP against LTL properties involving frequency operators [BDL12] also allows for an automata-theoretic approach, via deterministic generalized Rabin mean-payoff automata (DGRMA) [FKK15].

LTL synthesis can also be solved using the automata-theoretic approach. Although DRA and DGRA transformed into games can be used here, the algorithms for the resulting Rabin games [PP06] are not very efficient in practice. In contrast, DPA may be larger, but in this setting they are the automata of choice due to the good practical performance of parity-game solvers [FL09, ML16, JBB+17].

Types of Translations. The translations of LTL to NBA, e.g., [VW86], are typically "semantic" in the sense that each state is given by a set of logical formulae and the language of the state can be captured in terms of semantics of these formulae. In contrast, the determinization of Safra [Saf88] or its improvements [Pit06,Sch09, TD14,FL15] are not "semantic" in the sense that they ignore the structure and produce trees as the new states that, however, lack the logical interpretation. As a result, if we apply Safra's determinization on semantically created NBA, we obtain DRA that lack the structure and, moreover, are unnecessarily large since the construction cannot utilize the original structure. In contrast, the

\footnotetext{
${ }^{1}$ Note that every DGRA can be written as a Muller automaton on the same state space with an exponentially-sized acceptance condition, and DPA are a special case of DRA and thus DGRA.
} 
recent works [KE12,KLG13, EK14, KV15,SEJK16, EKRS17, MS17, KV17] provide "semantic" constructions, often producing smaller automata. Furthermore, various transformations such as degeneralization [KE12], index appearance record [KMWW17] or determinization of limit-deterministic automata [EKRS17] preserve the semantic description, allowing for further optimizations of the resulting automata.

Our Contribution. While all previous versions of Rabinizer [GKE12,KLG13, KK14] featured only the translation LTL $\rightarrow$ DGRA $\rightarrow$ DRA, Rabinizer 4 now implements all the translations depicted by the solid arrows in Fig. 1. It improves all these translations, both algorithmically and implementation-wise, and moreover, features the first implementation of the translation of a frequency extension of LTL [FKK15].

Further, in order to utilize the resulting automata for verification, we provide our own distribution ${ }^{2}$ of the PRISM model checker [KNP11], which allows for model checking MDP against LTL using not only DRA and DGRA, but also using LDBA and against frequency LTL using DGRMA. Finally, the tool can turn the produced DPA into parity games between the players with input and output variables. Therefore, when linked to parity-game solvers, Rabinizer 4 can be also used for LTL synthesis.

Rabinizer 4 is freely available at http://rabinizer.model.in.tum.de together with an on-line demo, visualization, usage instructions and examples.

\section{Functionality}

We recall that the previous version Rabinizer 3 has the following functionality:

- It translates LTL formulae into equivalent DGRA or DRA.

- It is linked to PRISM, allowing for probabilistic verification using DGRA (previously PRISM could only use DRA).

\subsection{Translations}

Rabinizer 4 inputs formulae of LTL and outputs automata in the standard HOA format $[\mathrm{BBD}+15]$, which is used, e.g., as the input format in PRISM. Automata in the HOA format can be directly visualized, displaying the "semantic" description of the states. Rabinizer 4 features the following command-line tools for the respective translations depicted as the solid arrows in Fig. 1:

ltl2dgra and lt12dra correspond to the original functionality of Rabinizer 3, i.e., they translate LTL (now with the extended syntax, including all common temporal operators) to DGRA and DRA [EK14], respectively.

\footnotetext{
${ }^{2}$ Merging these features into the public release of PRISM as well as linking the new version of Rabinizer is subject to current collaboration with the authors of PRISM.
} 
ltl2ldgba and ltl2ldba translate LTL to LDGBA using the construction of [SEJK16] and to LDBA, respectively. The latter is our modification of the former, which produces smaller automata than chaining the former with the standard degeneralization.

ltl2dpa translates LTL to DPA using two modes:

- The default mode uses the translation to LDBA, followed by a LDBAto-DPA determinization [EKRS17] specially tailored to LDBA with the "semantic" labelling of states, avoiding additional exponential blow-up of the resulting automaton.

- The alternative mode uses the translation to DRA, followed by our improvement of the index appearance record of [KMWW17].

flt12dgrma translates the frequency extension of $\mathrm{LTL}_{\backslash \mathbf{G U}}$, i.e. $\mathrm{LTL}_{\backslash \mathbf{G U}}[\mathrm{KLG} 13$ ] with $\mathbf{G}^{\sim \rho}$ operator $^{3}$, to DGRMA using the construction of [FKK15].

\section{$2.2 \quad$ Verification and Synthesis}

The resulting automata can be used for model checking probabilistic systems and for LTL synthesis. To this end, we provide our own distribution of the probabilistic model checker PRISM as well as a procedure transforming automata into games to be solved.

Model checking: PRISM distribution. For model checking Markov chains and Markov decision processes, PRISM [KNP11] uses DRA and recently also more efficient DGRA [CGK13,KK14]. Our distribution, which links Rabinizer, additionally features model checking using the LDBA [SEJK16, SK16] that are created by our ltl2ldba.

Further, the distribution provides an implementation of frequency $\operatorname{LTL}_{\backslash \mathbf{G U}}$ model checking, using DGRMA. To the best of our knowledge, there are no other implemented procedures for logics with frequency. Here, techniques of linear programming for multi-dimensional mean-payoff satisfaction [CKK15] and the model-checking procedure of [FKK15] are implemented and applied.

Synthesis: Games. The automata-theoretic approach to LTL synthesis requires to transform the LTL formula into a game of the input and output players. We provide this transformer and thus an end-to-end LTL synthesis solution, provided a respective game solver is linked. Since current solutions to Rabin games are not very efficient we implemented a transformation of DPA into parity games and a serialization to the format of PG Solver [FL09]. Due to the explicit serialization, we foresee the main use in quick prototyping.

${ }^{3}$ The frequential globally construct [BDL12,BMM14] $\mathbf{G}^{\sim \rho} \varphi$ with $\sim \in$ $\{\geq,>, \leq,<\}, \rho \in[0,1]$ intuitively means that the fraction of positions satisfying $\varphi$ satisfies $\sim \rho$. Formally, the fraction on an infinite run is defined using the long-run average [BMM14]. 


\section{Optimizations, Implementation, and Evaluation}

Compared to the theoretical constructions and previous implementations, there are numerous improvements, heuristics, and engineering enhancements. We evaluate the improvements both in terms of the size of the resulting automaton as well as the running time. When comparing with respect to the original Rabinizer functionality, we compare our implementation ltl2dgra to the previous version Rabinizer 3.1, which is already a significantly faster [EKS16] re-implementation of the official release Rabinizer 3 [KK14]. All of the benchmarks have been executed on a host with i7-4700MQ CPU (4x2.4 GHz), running Linux 4.9.0-5-amd64 and the Oracle JRE 9.0.4+11 JVM. Due to the start-up time of JVM, all times below $2 \mathrm{~s}$ are denoted by $<2$ and not specified more precisely. All experiments were given a time-out of $900 \mathrm{~s}$ and mem-out of $4 \mathrm{~GB}$, denoted by - .

Algorithmic improvements and heuristics for each of the translations:

lt12dgra and lt12dra. These translations create a master automaton monitoring the satisfaction of the given formula and a dedicated slave automaton for each subformula of the form $\mathbf{G} \psi$ [EK14]. We (i) simplify several classes of slaves and (ii) "suspend" (in the spirit of [BBDL+13]) some so that they appear in the final product only in some states. The effect on the size of the state space is illustrated in Table 1 on a nested formula. Further, (iii) the acceptance condition is considered separately for each strongly connected component (SCC) and then combined. On a concrete example of Table 2, the automaton for $i=8$ has 31 atomic propositions, whereas the number of atomic propositions relevant in each component of the master automaton is constant, which we utilize and thus improve performance on this family both in terms of size and time.

lt12ldba. This translation is based on breakpoints for subformulae of the form $\mathbf{G} \psi$. We provide a heuristic that avoids breakpoints when $\psi$ is a safety or co-safety subformula, see Table 3 .

Besides, we add an option to generate a non-deterministic initial component for the LDBA instead of a deterministic one. Although the LDBA is then no more suitable for quantitative probabilistic model checking, it still is for qualitative model checking. At the same time, it can be much smaller, see Table 4 which shows a significant improvement on the particular formula.

ltl2dpa. Both modes inherit the improvements of the respective ltl2ldba and lt12dgra translations. Further, since complementing DPA is trivial, we can run in parallel both the translation of the input formula and of its negation, returning the smaller of the two results. Finally, we introduce several heuristics to optimize the treatment of safety subformulae of the input formula.

dra2dpa. The index appearance record of [KMWW17] keeps track of a permutation (ordering) of Rabin pairs. To do so, all ties between pairs have to be resolved. In our implementation, we keep a pre-order instead, where irrelevant 
ties are not resolved. Consequently, it cannot happen that an irrelevant tie is resolved in two different ways like in [KMWW17], thus effectively merging such states.

Table 1. Effect of simplifications and suspension for ltl2dgra on the formulae $\psi_{i}=$ $\mathbf{G} \phi_{i}$ where $\phi_{1}=a_{1}, \phi(i)=\left(a_{i} \mathbf{U}\left(\mathbf{X} \phi_{i-1}\right)\right)$, and $\psi_{i}^{\prime}=\mathbf{G} \phi_{i}^{\prime}$ where $\phi_{1}^{\prime}=a_{1}, \phi_{1}^{\prime}=$ $\left(\phi_{i-1}^{\prime} \mathbf{U}\left(\mathbf{X}^{i} a_{i}\right)\right.$, displaying execution time in seconds/\#states.

\begin{tabular}{l|l|l|l|l|l}
\hline & $\psi_{2}$ & $\psi_{3}$ & $\psi_{4}$ & $\psi_{5}$ & $\psi_{6}$ \\
\hline Rabinizer 3.1 [EKS16] & $<2 / 4$ & $<2 / 16$ & $<2 / 73$ & $3 / 332$ & $60 / 1463$ \\
\hline lt12dgra & $<2 / 3$ & $<2 / 7$ & $<2 / 35$ & $3 / 199$ & $13 / 1155$ \\
\hline & $\psi_{2}^{\prime}$ & $\psi_{3}^{\prime}$ & $\psi_{4}^{\prime}$ & $\psi_{5}^{\prime}$ & $\psi_{6}^{\prime}$ \\
\hline Rabinizer 3.1 [EKS16] & $<2 / 4$ & $<2 / 16$ & $2 / 104$ & $128 / 670$ & - \\
\hline lt12dgra & $<2 / 3$ & $<2 / 10$ & $<2 / 38$ & $7 / 175$ & $239 / 1330$
\end{tabular}

Table 2. Effect of computing acceptance sets per SCC on formulae $\psi_{1}=x_{1} \wedge \phi_{1}$, $\psi_{2}=\left(x_{1} \wedge \phi_{1}\right) \vee\left(\neg x_{1} \wedge \phi_{2}\right), \psi_{3}=\left(x_{1} \wedge x_{2} \wedge \phi_{1}\right) \vee\left(\neg x_{1} \wedge x_{2} \wedge \phi_{2}\right) \vee\left(x_{1} \wedge \neg x_{2} \wedge \phi_{3}\right), \ldots$, where $\phi_{i}=\mathbf{X G}\left(\left(a_{i} \mathbf{U} b_{i}\right) \vee\left(c_{i} \mathbf{U} d_{i}\right)\right)$, displaying execution time in seconds/\#acceptance sets.

\begin{tabular}{l|l|l|l|l|l|l|l}
\hline & $\psi_{1}$ & $\psi_{2}$ & $\psi_{3}$ & $\psi_{4}$ & $\psi_{5}$ & $\ldots$ & $\psi_{8}$ \\
\hline Rabinizer 3.1 [EKS16] & $<2 / 2$ & $<2 / 7$ & $<2 / 19$ & - & - & & - \\
\hline lt12dgra & $<2 / 1$ & $<2 / 1$ & $<2 / 1$ & $<2 / 1$ & $<2 / 1$ & & $<2 / 1$ \\
\hline
\end{tabular}

Table 3. Effect of break-point elimination for ltl2ldba on safety formulae $s(n, m)=$ $\bigwedge_{i=1}^{n} \mathbf{G}\left(a_{i} \vee \mathbf{X}^{m} b_{i}\right)$ and for ltl2ldgba on liveness formulae $l(n, m)=\bigwedge_{i=1}^{n} \mathbf{G F}\left(a_{i} \wedge\right.$ $\mathbf{X}^{m} b_{i}$ ), displaying \#states (\#Büchi conditions)

\begin{tabular}{l|l|l|l|l|l|l|l|l}
\hline & $s(1,3)$ & $s(2,3)$ & $s(3,3)$ & $s(4,3)$ & $s(1,4)$ & $s(2,4)$ & $s(3,4)$ & $s(4,4)$ \\
\hline [SEJK16] & $20(1)$ & $400(2)$ & $8 \cdot 10^{3}(3)$ & $16 \cdot 10^{4}(4)$ & $48(1)$ & $2304(2)$ & $110592(3)$ & - \\
\hline lt12ldba & $8(1)$ & $64(1)$ & $512(1)$ & $4096(1)$ & $16(1)$ & $256(1)$ & $4096(1)$ & $65536(1)$ \\
\hline & $l(1,1)$ & $l(2,1)$ & $l(3,1)$ & $l(4,1)$ & $l(1,4)$ & $l(2,4)$ & $l(3,4)$ & $l(4,4)$ \\
\hline [SEJK16] & $3(1)$ & $9(2)$ & $27(3)$ & $81(4)$ & $10(1)$ & $100(2)$ & $10^{3}(3)$ & $10^{4}(4)$ \\
\hline ltl2ldgba & $3(1)$ & $5(2)$ & $9(3)$ & $17(4)$ & $3(1)$ & $5(2)$ & $9(3)$ & $17(4)$ \\
\hline
\end{tabular}

Table 4. Effect of non-determinism of the initial component for lt12ldba on formulae $f(i)=\mathbf{F}\left(a \wedge \mathbf{X}^{i} \mathbf{G} b\right)$, displaying \#states (\#Büchi conditions)

\begin{tabular}{l|l|l|l|l|l|r}
\hline & $f(1)$ & $f(2)$ & \multicolumn{1}{|l|}{$f(3)$} & \multicolumn{1}{l|}{$f(4)$} & \multicolumn{1}{l|}{$f(5)$} & \multicolumn{1}{l|}{$f(6)$} \\
\hline [SEJK16] & $4(1)$ & $6(1)$ & $10(1)$ & $18(1)$ & $34(1)$ & $66(1)$ \\
\hline lt12ldba & $2(1)$ & $3(1)$ & $4(1)$ & $5(1)$ & $6(1)$ & $7(1)$ \\
\hline
\end{tabular}


Table 5. Comparison of the average performance with the previous version of Rabinizer. The statistics are taken over a set of 200 standard formulae [KMS18] used, e.g., in [BKS13,EKS16], run in a batch mode for both tools to eliminate the effect of the JVM start-up overhead.

\begin{tabular}{l|l|l|l}
\hline Tool & Avg \# states & Avg \# acc. sets & Avg runtime \\
\hline Rabinizer 3.1 [EKS16] & 6.3 & 6.7 & 0.23 \\
\hline ltl2dgra & 6.2 & 4.4 & 0.12 \\
\hline
\end{tabular}

Implementation. The main performance bottleneck of the older implementations is that explicit data structures for the transition system are not efficient for larger alphabets. To this end, Rabinizer 3.1 provided symbolic (BDD) representation of states and edge labels. On the top, Rabinizer 4 represents the transition function symbolically, too.

Besides, there are further engineering improvements on issues such as storing the acceptance condition only as a local edge labelling, caching, data-structure overheads, SCC-based divide-and-conquer constructions, or the introduction of parallelization for batch inputs.

Average Performance Evaluation. We have already illustrated the improvements on several hand-crafted families of formulae. In Tables 1 and 2 we have even seen the respective running-time speed-ups. As the basis for the overall evaluation of the improvements, we use some established datasets from literature, see [KMS18], altogether two hundred formulae. The results in Table 5 indicate that the performance improved also on average among the more realistic formulae.

\section{Conclusion}

We have presented Rabinizer 4, a tool set to translate LTL to various deterministic automata and to use them in probabilistic model checking and in synthesis. The tool set extends the previous functionality of Rabinizer, improves on previous translations, and also gives the very first implementations of frequency LTL translation as well as model checking. Finally, the tool set is also more userfriendly due to richer input syntax, its connection to PRISM and PG Solver, and the on-line version with direct visualization, which can be found at http:// rabinizer.model.in.tum.de.

\section{References}

[BBD+15] Babiak, T., et al.: The hanoi omega-automata format. In: Kroening, D., Păsăreanu, C.S. (eds.) CAV 2015. LNCS, vol. 9206, pp. 479-486. Springer, Cham (2015). https://doi.org/10.1007/978-3-319-21690-4_31 
[BBDL+13] Babiak, T., Badie, T., Duret-Lutz, A., Křetínský, M., Strejček, J.: Compositional approach to suspension and other improvements to LTL translation. In: Bartocci, E., Ramakrishnan, C.R. (eds.) SPIN 2013. LNCS, vol. 7976, pp. 81-98. Springer, Heidelberg (2013). https://doi.org/10.1007/ 978-3-642-39176-7_6

[BDK+17] Blahoudek, F., Duret-Lutz, A., Klokočka, M., Křetínský, M., Strejček, J.: Seminator: a tool for semi-determinization of omega-automata. In: LPAR, pp. 356-367 (2017)

[BDL12] Bollig, B., Decker, N., Leucker, M.: Frequency linear-time temporal logic. In: TASE, pp. 85-92 (2012)

[BKŘS12] Babiak, T., Křetínský, M., Řehák, V., Strejček, J.: LTL to Büchi automata translation: fast and more deterministic. In: Flanagan, C., König, B. (eds.) TACAS 2012. LNCS, vol. 7214, pp. 95-109. Springer, Heidelberg (2012). https://doi.org/10.1007/978-3-642-28756-5_8

[BKS13] Blahoudek, F., Křetínský, M., Strejček, J.: Comparison of LTL to deterministic rabin automata translators. In: McMillan, K., Middeldorp, A., Voronkov, A. (eds.) LPAR 2013. LNCS, vol. 8312, pp. 164-172. Springer, Heidelberg (2013). https://doi.org/10.1007/978-3-642-45221-5_12

[BMM14] Bouyer, P., Markey, N., Matteplackel, R.M.: Averaging in LTL. In: Baldan, P., Gorla, D. (eds.) CONCUR 2014. LNCS, vol. 8704, pp. 266-280. Springer, Heidelberg (2014). https://doi.org/10.1007/978-3-662-445846_19

[CGK13] Chatterjee, K., Gaiser, A., Křetínský, J.: Automata with generalized rabin pairs for probabilistic model checking and LTL synthesis. In: Sharygina, N., Veith, H. (eds.) CAV 2013. LNCS, vol. 8044, pp. 559-575. Springer, Heidelberg (2013). https://doi.org/10.1007/978-3-642-397998_37

[CKK15] Chatterjee, K., Komárková, Z., Křetínský, J.: Unifying two views on multiple mean-payoff objectives in Markov decision processes. In: LICS, pp. 244-256 (2015)

[CY88] Courcoubetis, C., Yannakakis, M.: Verifying temporal properties of finitestate probabilistic programs. In: FOCS, pp. 338-345 (1988)

[DLLF+16] Duret-Lutz, A., Lewkowicz, A., Fauchille, A., Michaud, T., Renault, É., $\mathrm{Xu}, \mathrm{L}$.: Spot $2.0-\mathrm{a}$ framework for LTL and $\omega$-automata manipulation. In: Artho, C., Legay, A., Peled, D. (eds.) ATVA 2016. LNCS, vol. 9938, pp. 122-129. Springer, Cham (2016). https://doi.org/10.1007/978-3-31946520-3_8

[EH00] Etessami, K., Holzmann, G.J.: Optimizing Büchi automata. In: Palamidessi, C. (ed.) CONCUR 2000. LNCS, vol. 1877, pp. 153-168. Springer, Heidelberg (2000). https://doi.org/10.1007/3-540-44618-4_13

[EK14] Esparza, J., Křetínský, J.: From LTL to deterministic automata: a safraless compositional approach. In: Biere, A., Bloem, R. (eds.) CAV 2014. LNCS, vol. 8559, pp. 192-208. Springer, Cham (2014). https://doi.org/ 10.1007/978-3-319-08867-9_13

[EKRS17] Esparza, J., Křetínský, J., Raskin, J.-F., Sickert, S.: From LTL and limit-deterministic Büchi automata to deterministic parity automata. In: Legay, A., Margaria, T. (eds.) TACAS 2017. LNCS, vol. 10205, pp. 426-442. Springer, Heidelberg (2017). https://doi.org/10.1007/9783-662-54577-5_25 
[EKS16] Esparza, J., Kretínský, J., Sickert, S.: From LTL to deterministic automata - a safraless compositional approach. Formal Methods Syst. Des. 49(3), 219-271 (2016)

[FKK15] Forejt, V., Krčál, J., Křetínský, J.: Controller synthesis for MDPs and frequency LTL $\backslash$ GU. In: LPAR, pp. 162-177 (2015)

[FL09] Friedmann, O., Lange, M.: Solving parity games in practice. In: Liu, Z., Ravn, A.P. (eds.) ATVA 2009. LNCS, vol. 5799, pp. 182-196. Springer, Heidelberg (2009). https://doi.org/10.1007/978-3-642-04761-9_15

[FL15] Fisman, D., Lustig, Y.: A modular approach for büchi determinization. In: CONCUR, pp. 368-382 (2015)

[GKE12] Gaiser, A., Křetínský, J., Esparza, J.: Rabinizer: small deterministic automata for $\operatorname{LTL}(\mathbf{F}, \mathbf{G})$. In: Chakraborty, S., Mukund, M. (eds.) ATVA 2012. LNCS, pp. 72-76. Springer, Heidelberg (2012). https://doi.org/10. 1007/978-3-642-33386-6_7

[GL02] Giannakopoulou, D., Lerda, F.: From states to transitions: improving translation of LTL formulae to Büchi automata. In: Peled, D.A., Vardi, M.Y. (eds.) FORTE 2002. LNCS, vol. 2529, pp. 308-326. Springer, Heidelberg (2002). https://doi.org/10.1007/3-540-36135-9_20

[GO01] Gastin, P., Oddoux, D.: Fast LTL to Büchi automata translation. In: Berry, G., Comon, H., Finkel, A. (eds.) CAV 2001. LNCS, vol. 2102, pp. 53-65. Springer, Heidelberg (2001). https://doi.org/10.1007/3-54044585-4_6. http://www.lsv.ens-cachan.fr/ gastin/ltl2ba/

[HLS+15] Hahn, E.M., Li, G., Schewe, S., Turrini, A., Zhang, L.: Lazy probabilistic model checking without determinisation. In: CONCUR. LIPIcs, vol. 42, pp. 354-367 (2015)

[JBB+17] Jacobs, S., Basset, N., Bloem, R., Brenguier, R., Colange, M., Faymonville, P., Finkbeiner, B., Khalimov, A., Klein, F., Michaud, T., Pérez, G.A., Raskin, J.-F., Sankur, O., Tentrup, L.: The 4th reactive synthesis competition (SYNTCOMP 2017): benchmarks, participants \& results. CoRR, abs/1711.11439 (2017)

[KE12] Křetínský, J., Esparza, J.: Deterministic automata for the (F,G)-fragment of LTL. In: Madhusudan, P., Seshia, S.A. (eds.) CAV 2012. LNCS, vol. 7358, pp. 7-22. Springer, Heidelberg (2012). https://doi.org/10.1007/9783-642-31424-7_7

[KK14] Komárková, Z., Křetínský, J.: Rabinizer 3: safraless translation of LTL to small deterministic automata. In: Cassez, F., Raskin, J.-F. (eds.) ATVA 2014. LNCS, vol. 8837, pp. 235-241. Springer, Cham (2014). https://doi. org/10.1007/978-3-319-11936-6_17

[Kle] Klein, J.: ltl2dstar - LTL to deterministic Streett and Rabin automata. http://www.ltl2dstar.de/

[KLG13] Křetínský, J., Garza, R.L.: Rabinizer 2: Small Deterministic Automata for LTL \GU. In: Van Hung, D., Ogawa, M. (eds.) ATVA 2013. LNCS, vol. 8172, pp. 446-450. Springer, Cham (2013). https://doi.org/10.1007/ 978-3-319-02444-8_32

[KMS18] Křetínský, J., Meggendorfer, T., Sickert, S.: LTL store: repository of LTL formulae from literature and case studies. CoRR, abs/1807.03296 (2018)

[KMWW17] Křetínský, J., Meggendorfer, T., Waldmann, C., Weininger, M.: Index appearance record for transforming rabin automata into parity automata. In: Legay, A., Margaria, T. (eds.) TACAS 2017. LNCS, vol. 10205, pp. 443-460. Springer, Heidelberg (2017). https://doi.org/10.1007/9783-662-54577-5_26 
[KNP11] Kwiatkowska, M., Norman, G., Parker, D.: PRISM 4.0: verification of probabilistic real-time systems. In: Gopalakrishnan, G., Qadeer, S. (eds.) CAV 2011. LNCS, vol. 6806, pp. 585-591. Springer, Heidelberg (2011). https://doi.org/10.1007/978-3-642-22110-1_47

[KV15] Kini, D., Viswanathan, M.: Limit deterministic and probabilistic automata for LTL $\backslash$ GU. In: Baier, C., Tinelli, C. (eds.) TACAS 2015. LNCS, vol. 9035, pp. 628-642. Springer, Heidelberg (2015). https://doi. org/10.1007/978-3-662-46681-0_57

[KV17] Kini, D., Viswanathan, M.: Optimal translation of LTL to limit deterministic automata. In: Legay, A., Margaria, T. (eds.) TACAS 2017. LNCS, vol. 10206, pp. 113-129. Springer, Heidelberg (2017). https://doi.org/10. 1007/978-3-662-54580-5_7

[ML16] Meyer, P.J., Luttenberger, M.: Solving mean-payoff games on the GPU. In: Artho, C., Legay, A., Peled, D. (eds.) ATVA 2016. LNCS, vol. 9938, pp. 262-267. Springer, Cham (2016). https://doi.org/10.1007/978-3-31946520-3_17

[MS17] Müller, D., Sickert, S.: LTL to deterministic Emerson-Lei automata. In: GandALF, pp. 180-194 (2017)

[Pit06] Piterman, N.: From nondeterministic Büchi and Streett automata to deterministic parity automata. In: LICS, pp. 255-264 (2006)

[Pnu77] Pnueli, A.: The temporal logic of programs. In: FOCS, pp. $46-57$ (1977)

[PP06] Piterman, N., Pnueli, A.: Faster solutions of Rabin and Streett games. In: LICS, pp. 275-284 (2006)

[Saf88] Safra, S.: On the complexity of omega-automata. In: FOCS, pp. 319-327 (1988)

[SB00] Somenzi, F., Bloem, R.: Efficient Büchi automata from LTL formulae. In: Emerson, E.A., Sistla, A.P. (eds.) CAV 2000. LNCS, vol. 1855, pp. 248263. Springer, Heidelberg (2000). https://doi.org/10.1007/10722167_21

[Sch09] Schewe, S.: Tighter bounds for the determinisation of Büchi automata. In: de Alfaro, L. (ed.) FoSSaCS 2009. LNCS, vol. 5504, pp. 167-181. Springer, Heidelberg (2009). https://doi.org/10.1007/978-3-642-00596-1_13

[SEJK16] Sickert, S., Esparza, J., Jaax, S., Křetínský, J.: Limit-deterministic Büchi automata for linear temporal logic. In: Chaudhuri, S., Farzan, A. (eds.) CAV 2016. LNCS, vol. 9780, pp. 312-332. Springer, Cham (2016). https://doi.org/10.1007/978-3-319-41540-6_17

[SK16] Sickert, S., Křetínský, J.: MoChiBA: probabilistic LTL model checking using limit-deterministic Büchi automata. In: Artho, C., Legay, A., Peled, D. (eds.) ATVA 2016. LNCS, vol. 9938, pp. 130-137. Springer, Cham (2016). https://doi.org/10.1007/978-3-319-46520-3_9

[TD14] Tian, C., Duan, Z.: Buchi determinization made tighter. Technical report abs/1404.1436, arXiv.org (2014)

[VW86] Vardi, M.Y., Wolper, P.: An automata-theoretic approach to automatic program verification (preliminary report). In: LICS, pp. 332-344 (1986) 
Open Access This chapter is licensed under the terms of the Creative Commons Attribution 4.0 International License (http://creativecommons.org/licenses/by/4.0/), which permits use, sharing, adaptation, distribution and reproduction in any medium or format, as long as you give appropriate credit to the original author(s) and the source, provide a link to the Creative Commons license and indicate if changes were made.

The images or other third party material in this chapter are included in the chapter's Creative Commons license, unless indicated otherwise in a credit line to the material. If material is not included in the chapter's Creative Commons license and your intended use is not permitted by statutory regulation or exceeds the permitted use, you will need to obtain permission directly from the copyright holder.

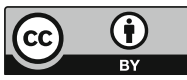

